\title{
Discrete and Continuous Wavelet Expansions
}

\author{
YURI DEM'YANOVICH \\ Parallel Algorithm Department \\ Saint-Petersburg State University \\ Saint Petersburg \\ RUSSIA \\ LE THI NHU BICH \\ College of Education \\ Hue University \\ VIETNAM
}

\begin{abstract}
This paper proposes a new approach to the construction of wavelet decomposition, which is suitable for processing a wide range of information flows. The proposed approach is based on abstract functions with values in linear topological spaces. It is defined by embedded spaces and their projections. The proposed approach allows for adaptive ways of decomposition for the initial flow depending on the speed changes of the last one. The initial information flows can be real number flows, flows of complex and p-adic numbers, as well as flows of (finite or infinite) vectors, matrices, etc. The result is illustrated with examples of splinewavelet decompositions of discrete flows, and also with the example of the decomposition of a continuous flow.
\end{abstract}

Key-Words: - wavelets, flows, decomposition, reconstruction, calibration relations

Received: April 16, 2021. Revised: January 3, 2022. Accepted: January 22, 2022. Published: February 23, 2022.

\section{Introduction}

The constant growth in the volume of numerical information flows stimulates further development and improvement employing their processing. The structure and nature of these flows depend substantially on areas of human activity (economics, medicine, technology, etc.), the means and methods of obtaining information, from the quality of communication channels and their processing speed.

Wavelet decomposition is one of the main means of the processing of numerical information flows. Its advantage over other processing methods consists of the fact that the original numerical flow is split into the main part, specifying (wavelet) part and non-essential part. This explains the widespread use of wavelet expansions in various fields of human activity. Let us give several examples of the application of these expansions in technology and medicine.

In research [1] the separate models for signal denoising with different ratio signal/noise were designed. The discrete wavelet decompositions were used. The result was applied to the computerized analysis of Lung Sound.

Paper [2] is devoted to the damage severity quantification of the brain by using a wavelet packet. The proposed technique shows significant benefits in compressing spatio-spectral patterns of multichannel signals in just a unified visual representation.

The timely and high-quality maintenance of electrical networks is a prerequisite for their troublefree operation. In work [3], complex wavelets are used to create an efficient algorithm for such processing. The proposed algorithm achieves higher accuracy with reduced training time in the classification of events than compared to the reported event classification methods.

As we can see from the above examples, currently, the main problem is having insufficient speed processing numerical information reaching huge volumes. To solve this problem, a significant advancement in the development of fast wavelet algorithms decomposition is needed.

To date, there are several studies on the theory of wavelets, among which special mentions are 
chilled works of I. Daubeshies [4], K. Chui [5], S. Mallat [6], Yu. N. Subbotin and Chernykh [7], I. Ya. Novikov, V. Yu. Protasov and M. A. Skopina [8]. Research in this direction also includes a series of modern works.

Cubic wavelets with two zero moments are obtained in work [9]. Five-diagonal splitting for cubic splines with six zero moments on the segment was obtained in [10]. Paper [11] deals with the structural issues that concern wavelet frames and their dual frames. In paper [12] the authors define the wavelet multiplier and Landau-Pollak-Slepian operators on the Hilbert space. In paper [13] the wavelet optimized finite difference B-spline polynomial chaos method is proposed. The method is applied to the solution of stochastic partial differential equations. In paper [14] the authors propose a highly efficient and accurate valuation method for exotic-style options based on the novel Shannon wavelet inverse Fourier technique (SWIFT).

A new structure based on wavelet neural networks, deep architecture and the Extreme Learning Machine is proposed in paper [15]. The proposed method is based on the Extreme Learning Machine Auto-Encoder with a deep learning structure and a composite wavelet activation function used in the hidden nodes. Paper [16] presents the rates of uniform strong consistency of wavelet estimation for the nonparametric function in the sup-norm loss by introducing an empirical process approach. Paper [17] presents a new construction of the homogeneous Dirichlet wavelet basis on the unit interval. In work [18], a suitable mother wavelet is selected for effective crack detection in the beam. Paper [19] proposes a parameter identification method of fractionalorder time delay system based on the Legendre wavelet.

These studies mainly reflect the classic approach to wavelets, which is based on various variants of the Fourier transform, applied to the multiple-scale ratio to obtain a scaling function and ultimately wavelet decomposition.

However, the practice of processing numeric flows required expanding the framework of the classical theory. W.Sveldens constructed a lifting scheme for an area that is not invariant relative to the shift. The concept of non-stationary wavelets, introduced by I.Ya. Novikov, also led to the expansion the framework of the mentioned theory. The need to significantly speed up computations was faced with great theoretical difficulties that arose on the path of the development of the classical approach to the wavelet expansions.
The purpose of this paper is to consider another spline-wavelet scheme decomposition. The proposed approach is based on abstract functions with values in linear topological spaces. It is defined by embedded spaces and their projections. The proposed approach allows for adaptive ways of decomposition for the initial flow depending on the speed changes of the last one. As initial information flows can be real number flows, flows of complex and p-adic numbers, as well as flows of (finite or infinite) vectors, matrices, etc. The result is illustrated with examples of spline-wavelet decompositions of discrete flows, and also on the example of the decomposition of a continuous flow.

The results obtained along this path lead to the simple formulas decomposition and reconstruction. In view of this, there is a significant acceleration of computations. In the reviewed practical examples in the case of rapidly changing flows, the computation time is greatly reduced.

\section{Auxiliary Assertion and Results}

Let $\Omega$ and $\widetilde{\Omega}$ be topological vector spaces. We denote conjugate spaces of linear continuous functionals by $\Omega^{*}$ and $\widetilde{\Omega}^{*}$ accordingly. Let $(c, f)_{\Omega}$ be the value, which is the result of the action of the functional $c \in \Omega^{*}$ on the element $f \in \Omega$.

Analogously by $(\tilde{a}, \tilde{f})_{\tilde{\Omega}} \quad$ we denote the result of the application of the functional $\tilde{a} \in \widetilde{\Omega^{*}}$ to element $\tilde{f} \in \widetilde{\Omega}$.

Let $T$ be an interval $(\alpha, \beta)$. In what follows, we consider abstract functions $F(t), t \in T=(\alpha, \beta)$, with values in some vector space $H$. If the closed linear shell of values for the function $F(t)$ coincides with the space $H$, then the function is called a complete function.

Lemma 1. If $f: T \rightarrow H$ is a complete function, then for any nonzero functional $g$ from the conjugate space $H^{*}$ there is a point

$$
t=t_{0} \in T \text { such that }\left(g, f\left(t_{0}\right)\right) \neq 0 \text {. }
$$

Proof. Proof by contradiction. Let $g$ be an arbitrary nonzero element belonging $H^{*}$. We suppose that $(g, f(t))=0 \forall t \in T$. By completeness of $f(t)$ we have $C l_{p}\{f(t) \mid t \epsilon T\}$. Taking into account the continuity of the functional $g$, we deduce that $g=0$.This contradiction concludes the proof.

Let $\omega(t)$ and $\widetilde{\omega}(t)$ be complete functions of the parameter $t \epsilon T$ with values in vector spaces $\Omega$ and $\widetilde{\Omega}$ respectively.

Consider vector spaces 
$S=\left\{u \mid u=u(t)=(c, \omega(t))_{\Omega}\right\}$,

$\widetilde{S}=\left\{\tilde{u} \mid \tilde{u}=\tilde{u}(t)=(\widetilde{a}, \widetilde{\omega}(t))_{\widetilde{\Omega}}\right\}$.

The fact that these spaces are vector follows from easily verifiable properties: from additivity and homogeneity with respect to operations of addition and multiplication by a value.

Lemma 2. The following statements are true.

1. For function $u \in S$ there is only one element $c \in \Omega^{*}$, for which representation $u(t)=(c, \omega(t))_{\Omega}$ is true.

2. For function $\tilde{u} \in \tilde{S}$ there is only one element $\tilde{a} \in \widetilde{\Omega}^{*}$, for which representation $\tilde{u}(t)=(\tilde{a}, \widetilde{\omega}(t))_{\widetilde{\Omega}}$ is true.

Proof. Since both statements of the lemma are stated under the same conditions with respect to the spaces $S$ and $\tilde{S}$, then it suffices to prove only the first of them.

We will prove by contradiction. Let there be elements $c^{\prime}, c^{\prime \prime} \in S, c^{\prime} \neq c^{\prime \prime}$, for which

$$
\left(c^{\prime}, \omega(t)\right)_{\Omega} \equiv\left(c^{\prime \prime}, \omega(t)\right)_{\Omega} \forall t \in T .
$$

We put $b=c^{\prime \prime}-c^{\prime}$. According to (1), we find point $t_{0} \in T$ such that

$$
\left(b, \omega\left(t_{0}\right)\right)_{\Omega} \neq 0 .
$$

Relation (3) contradicts formula (2). The received contradiction proves the first assertion of the lemma.

The second statement turns out to be similar. This concludes the proof.

Theorem 1. Let $\mathcal{P}$ be a linear operator

$$
\mathcal{P}: \Omega \rightarrow \widetilde{\Omega} \text {. }
$$

If the formula

$$
\widetilde{\omega}(t) \equiv \mathcal{P} \omega(t) \forall t \epsilon T
$$

is right, then the space $\tilde{S}$ is embedded in $S$,

$$
\tilde{S} \subset S \text {. }
$$

Proof. By (4) we have

$$
\mathcal{P}^{*}: \Omega^{*} \leftarrow \widetilde{\Omega}^{*}
$$

For each element $\tilde{u} \in \widetilde{S}$ we find $\tilde{a} \in \widetilde{\Omega}^{*}$ so that $\tilde{u}(t)=(\tilde{a}, \widetilde{\omega}(t))_{\widetilde{\Omega}}$. Taking into account formula (5), we have

$$
\tilde{u}(t)=(\tilde{a}, \mathcal{P} \omega(t))_{\widetilde{\Omega}} .
$$

By (8) we reduce

$\tilde{u}(t)=\left(\mathcal{P}^{*} \tilde{a}, \omega(t)\right)_{\Omega}=(c, \omega(t))_{\Omega}$,

where $c=\mathcal{P}^{*} \tilde{a} \in \Omega^{*}$. Thus, $\tilde{u}$ is an element of space $S$. Formula (6) has been established. This concludes the proof.

Let the space $\widetilde{S}$ be embedded in the space $\tilde{S}$, that is, relation (6) holds. Consider a projecting operator $P_{0}$, which projects the space $S$ onto $\widetilde{S}$, $P_{0}: S \rightarrow \widetilde{S}$.This means that for the element $u \in S$ there are only elements $c \in \Omega^{*}$ and $\tilde{a} \in \widetilde{\Omega}^{*}$ such that

$$
u(t)=(c, \omega(t))_{\Omega}, \tilde{u}(t)=(\tilde{a}, \widetilde{\omega}(t))_{\widetilde{\Omega}},
$$

$$
P_{0}\left[(c, \omega(\cdot))_{\Omega}\right](t)=(\tilde{a}, \widetilde{\omega}(t))_{\widetilde{\Omega}} .
$$

The element $\tilde{a} \in \widetilde{\Omega}^{*}$ is defined uniquely by $c \in \Omega^{*}$. Corresponding mapping $c \rightarrow \widetilde{a}$ is denoted by $\boldsymbol{Q}$,

$$
\tilde{a}=Q_{\text {c. }} \text {. }
$$

It is easy to see that $\boldsymbol{Q}$ is a linear operator acting from the space $\Omega^{*}$ into the space $\widetilde{\Omega}^{*}$,

$$
\text { Q: } \Omega^{*} \rightarrow \widetilde{\Omega}^{*} \text {. }
$$

Thus, operator $\mathcal{Q}$ is defined by projector $P_{0}$ of the space $S$ onto space $\widetilde{S}$ according to the formula

$$
(\boldsymbol{Q} c, \widetilde{\omega}(t))_{\widetilde{\Omega}} \equiv P_{0}\left[(c, \omega(\cdot))_{\Omega}\right](t) \quad \forall t \epsilon T .
$$

Theorem 2. For any element $u \in S, u(t)=$ $(c, \omega(t))_{\Omega}, c \in \Omega^{*}$, the relation

is true.

$$
P_{0}[u(\cdot)](t) \equiv\left(\mathcal{P}^{*} \boldsymbol{Q} \mathbf{c}, \omega(t)\right)_{\Omega} \quad \forall t \in T .
$$

Proof. From (7) and (9) we obtain

$$
\begin{aligned}
& P_{0}[u(\cdot)](t) \equiv(\boldsymbol{Q} \mathrm{c}, \widetilde{\omega}(t))_{\widetilde{\Omega}} \equiv \\
& \equiv(\boldsymbol{Q} \mathrm{c}, \mathcal{P} \omega(t))_{\widetilde{\Omega}} \quad \forall t \in T .
\end{aligned}
$$

Passing to the adjoint operator in (14), we obtain (13). This concludes the proof.

Let us introduce the operator $Q_{0}=I-P_{0}$. As a result of projection (9), we obtain the direct sum

where $\widetilde{S}=P_{0} S, W=Q_{0} S$.

$$
S=\widetilde{S}+W,
$$

Let $c \in \Omega^{*}$. We put

$$
b=c-\mathcal{P}^{*} \boldsymbol{Q} c .
$$

Theorem 3. The relations

are right. Here $\tilde{a}=\boldsymbol{Q}$ c.

$$
\begin{gathered}
Q_{0}[u(\cdot)](t)=u(t)-P_{0}[u(\cdot)](t)= \\
=(b, \omega(t))_{\Omega}, \\
c=\mathcal{P}^{*} \tilde{a}+b
\end{gathered}
$$

Proof. From (13) - (14) and (16) we have $Q_{0}[u(\cdot)](t)=u(t)-P_{0}[u(\cdot)](t)=(c, \omega(t))_{\Omega}-$ $\left(\mathcal{P}^{*} \boldsymbol{Q} \mathrm{c}, \omega(t)\right)_{\Omega}=(b, \omega(t))_{\Omega}$.

Thus, relation (17) is true. From (10) and (16) we obtain (18). This completes the proof.

Item $c \in \Omega^{*}$ is initial flow, item $\tilde{a}$ is main flow and $b$ is wavelet flow. Formulas (10), (16) represent decomposition formulas, and formula (18) represents reconstruction formula.

Theorem 4. The ratio

$$
\mathcal{Q P}^{*}=\mathcal{J}
$$

is right. Here $\mathcal{J}$ is the identity operator in space $\widetilde{\Omega}^{*}$.

Proof. Since operator $P_{0}$ is the projector onto space $\widetilde{S}$, then it acts as the identical operator on elements of this space. Thus, we have

$\equiv(\tilde{a}, \widetilde{\omega}(t))_{\widetilde{\Omega}} \forall \tilde{a} \epsilon \widetilde{\Omega}^{*} \forall t \epsilon T$.

$$
P_{0}\left[(\tilde{a}, \widetilde{\omega}(\cdot))_{\widetilde{\Omega}}\right](t) \equiv
$$

On the other hand, the rer for calcution of projection has the form (see (14)) 


$$
P_{0}\left[(c, \omega(\cdot))_{\Omega}\right](t) \equiv(\boldsymbol{Q} \mathrm{c}, \widetilde{\omega}(t))_{\widetilde{\Omega}} .
$$

Setting $c=\mathcal{P}^{*} \tilde{a}$, from (21) we find

$P_{0}\left[\left(\mathcal{P}^{*} \tilde{a}, \omega(\cdot)\right)_{\Omega}\right](t) \equiv$

$\left(\mathcal{Q P}^{*} \tilde{a}, \widetilde{\omega}(t)\right)_{\widetilde{\Omega}} \forall \tilde{a} \epsilon \widetilde{\Omega}^{*} \forall t \epsilon T$.

An obvious transformation of the left-hand side of the last relation gives the formula

$\equiv\left(\mathcal{Q P}^{*} \tilde{a}, \widetilde{\omega}(t)\right)_{\widetilde{\Omega}} \forall \tilde{a} \epsilon \widetilde{\Omega}^{*} \forall t \epsilon T$.

$$
P_{0}\left[(\tilde{a}, \mathcal{P} \omega(\cdot))_{\Omega}\right](t) \equiv
$$

Using property (5) on the left-hand side of formula (22), we obtain the equality of the left-hand side's relations (20) and (22). Therefore, the following formula is valid

$$
(\widetilde{a}, \widetilde{\omega}(t))_{\widetilde{\Omega}} \equiv\left(\mathcal{Q P}^{*} \widetilde{a}, \widetilde{\omega}(t)\right)_{\widetilde{\Omega}} \forall \tilde{a} \epsilon \widetilde{\Omega}^{*} .
$$

In view of the completeness of the abstract function $\widetilde{\omega}(t)$ from formula (23), we derive relation (19). This concludes the proof.

\section{On Embedding Spaces}

Let $\Omega$ be finite-dimensional spaces $\Omega=R^{M}$ of vectors $c=\left(c_{1}, c_{2}, \ldots, c_{M}\right)^{T}$ with the scalar product $\left(c^{\prime}, c^{\prime \prime}\right)_{R^{M}}=\sum_{i=1}^{M} c_{i}^{\prime} c_{i}^{\prime \prime}$.

Consider one more finite-dimensional space $\widetilde{\Omega}=R^{K}$ of vectors $\tilde{a}=\left(\tilde{a}_{1}, \tilde{a}_{2}, \ldots, \tilde{a}_{K}\right)^{T}$ with scalar product $\left(\tilde{a}^{\prime}, \tilde{a}^{\prime \prime}\right)_{R^{K}}=\sum_{i=1}^{K} \tilde{a}_{i}^{\prime} \tilde{a}_{i}^{\prime \prime}$. We consider the vector functions with a real argument. We suppose that the closure of the linear envelope of the set values of the functions coincides with the entire space, i.e. in this section we discuss complete functions.

Consider the complete functions $\omega(t)$ and $\widetilde{\omega}(t)$, $t \in T$, with values in the spaces $R^{M}$ and $R^{K}$, respectively.

We introduce linear spaces of functions by the relations

$S=\left\{u \mid u=(c, \omega(t))_{R^{M}} \forall c \in R^{M} \forall t \in T\right\}, \quad$ (24) $\tilde{S}=$ $\left\{\widetilde{u} \mid \widetilde{u}=(\widetilde{a}, \widetilde{\omega}(t))_{R^{K}} \forall \widetilde{a} \in R^{K} \forall t \epsilon T\right\}$. (25)

Theorem 5. If the number matrix $\mathcal{P}=\left(p_{i j}\right)$ of size $K \times M \quad$ has the property

$$
\widetilde{\omega}(t) \equiv \mathcal{P} \omega(t) \quad \forall t \in T,
$$

then the space (25) is embedded in the space (24), $\tilde{S} \subset S$.

Theorem 5 is a special case of Theorem 1 .

Example 1. Let $K=2, M=3$, so the matrix $\mathcal{P}=$ $\left(p_{i j}\right)$ has the size $2 \times 3$. Suppose that

$p_{11}=1, p_{12}=3, p_{13}=4, p_{21}=2, p_{22}=4, p_{23}=$ 6, $\omega(t)=\left(1, t, t^{2}\right)^{T}, \quad \widetilde{\omega}(t)=\left(1+3 t+4 t^{2}, 2+\right.$ $\left.4 t+6 t^{2}\right)^{T}$. We define the spaces

$S=\left\{c_{1}+c_{2} t+c_{3} t^{2} \mid \forall c=\left(c_{1}, c_{2}, c_{3}\right)^{T} \epsilon R^{3}\right\}$, $\widetilde{S}=\left\{\tilde{a}_{1}\left(1+3 t+4 t^{2}\right), \tilde{a}_{2}\left(2+4 t+6 t^{2}\right) \mid\right.$ $\left.\forall \tilde{a}=\left(\tilde{a}_{1}, \tilde{a}_{2}\right)^{T} \in R^{2}\right\} . \quad$ It is clear that the relation $\widetilde{\omega}(t) \equiv \mathcal{P} \omega(t) \forall t \in T \quad$ is right. By Theorem 1 the relation $\tilde{S}$ c $S$ is fulfilled.

Example 2. Let $K=3, M=2$, so the matrix $\mathcal{P}=$ $\left(p_{i j}\right)$ has the size $3 \times 2$. . Suppose that

$p_{11}=1, p_{12}=2, p_{21}=3, p_{22}=4, p_{31}=4, p_{32}=$ 6. We define $\omega(t)$ and $\widetilde{\omega}(t)$ by formulas $\omega(t)=$ $(1, t)^{T}, \widetilde{\omega}(t)=(1+2 t, 3+4 t, 4+6 t)^{T}$. Let us discuss the spaces

$S=\left\{c_{1}+c_{2} t \mid \forall c=\left(c_{1}, c_{2}\right)^{T} \epsilon R^{2}\right\}$, $\widetilde{S}=\left\{\tilde{a}_{1}(1+2 t), \tilde{a}_{2}(3+4 t), \tilde{a}_{3}(4+6 t) \mid\right.$ $\left.\forall \tilde{a}=\left(\tilde{a}_{1}, \tilde{a}_{2}, \tilde{a}_{3}\right)^{T} \epsilon R^{3}\right\}$.

Obviously, the relation $\widetilde{\omega}(t) \equiv \mathcal{P} \omega(t) \forall t \in T$ is right. By Theorem 1 the relation $\tilde{S}$ c $S$ is fulfilled.

\section{Zero-type Wavelets in Finite- Dimensional Spaces}

\subsection{Zero-order Splines}

Let $[\bar{\alpha}, \bar{\beta})$ be an interval of $R^{1}$. Consider a grid $\Xi_{M}: \bar{\alpha}=\xi_{0}<\xi_{1}<\cdots<\xi_{M-1}<\xi_{M}=\bar{\beta}$.

Let $J_{M}$ be the set $\{0,1,2, \ldots, M-1\}$. We introduce piecewise constant functions $\omega_{j}(t), t \in[\bar{\alpha}, \bar{\beta}), j \in J_{M}$, defined by the equalities

$$
\begin{aligned}
& \omega_{j}(t)=1 \text { for } t \epsilon\left[\xi_{j}, \xi_{j+1}\right), \\
& \quad \omega_{j}(t)=0 \text { for } t \bar{\epsilon}\left[\xi_{j}, \xi_{j+1}\right), j \in J_{M} .
\end{aligned}
$$

System $\omega(t)=\left\{\omega_{j}(t)\right\}_{j \in J_{M}}$ of the functions (26) is determined by the grid $\Xi_{M}$.

The functions $\omega_{j}(t)$ are coordinate splines of order zero.

For the space $\Omega$, we take space $R^{M}$ of real vectors

$$
c=\left(c_{0}, c_{1}, \ldots, c_{M-1}\right)^{T}
$$

with standard scalar product

$$
\left(c^{\prime}, c^{\prime \prime}\right)_{\Omega}=\sum_{i=0}^{M-1} c_{i}^{\prime} c_{i}^{\prime \prime} \quad \forall c^{\prime}, c^{\prime \prime} \in \Omega .
$$

In what follows, formula (27) is written in the form $c=\left(c_{i}\right)_{\left\{i \in J_{M}\right\}}^{T}$. Consider a linear space $S$ defined by the relation

$$
\begin{gathered}
S=\left\{u(t) \mid u(t)=\sum_{i \in J_{M}} c_{i} \omega_{i}(t)\right. \\
\left.\forall c=\left(c_{i}\right)_{\left\{i \in J_{M}\right\}}^{T} \in \Omega\right\} .
\end{gathered}
$$

This space is called \{lit generalized zero spline space on the grid $\Xi_{M}$, and the elements of this space are called splines of order zero.

Specifying the grid $\Xi_{M}$ uniquely determines the space $S$.

Note that for fixed $t \epsilon[\bar{\alpha}, \bar{\beta}]$ the sequence $\omega(t)$ contains one element equal to the unit. The rest of its elements are equal to zero. So this sequence is an element of space $\Omega$. So, $\omega(t)$ is an abstract 
function with values in the space $\Omega$. That is why the equivalence is valid

$$
\begin{aligned}
u(t) & =\sum_{i \in J_{M}} c_{i} \omega_{i}(t) \quad \forall c=\left(c_{i}\right)_{\left\{i \in J_{M}\right\}}^{T} \in \Omega \Leftrightarrow \\
\Leftrightarrow u(t) & =(c, \omega(t))_{\Omega} \forall c \in \Omega .
\end{aligned}
$$

In view of this, formula (28) can be written in the form

$$
S=\left\{u(t) \mid u(t)=(\mathrm{c}, \omega(t))_{\Omega} \forall c \in \Omega\right\} .
$$

\subsection{Embedded Space}

Let $K$ be a natural number, $\leq M$. We discuss the same half-open interval $[\bar{\alpha}, \bar{\beta})$. Consider a grid $\widetilde{\Xi}_{K}$, which is embedded in $\widetilde{\Xi}_{M}$,

$\tilde{\Xi}_{K}: \bar{\alpha}=\tilde{\xi}_{0}<\tilde{\xi}_{1}<\cdots<\tilde{\xi}_{K-1}<\tilde{\xi}_{K}=\bar{\beta}$.

Thus, for each $j \epsilon J_{K}$ we have $\tilde{\xi}_{j} \in \Xi_{M}$. Therefore, for each $j \epsilon J_{K}$ there is a single number $i \epsilon J_{M}$ such that $\tilde{\xi}_{j}=\xi_{i}$. We denote this mapping $\tilde{\mathcal{H}}$,

$$
\tilde{\varkappa}: J_{K} \rightarrow J_{M}, \quad i=\tilde{\varkappa}(j), \quad \tilde{\xi}_{j}=\xi \tilde{\varkappa}(j) .
$$

Let $J_{K}^{*}=\tilde{\mathcal{H}}\left(J_{K}\right)$. It is obvious that $J_{K}^{*} c J_{M}$. The unambiguous inverse mapping is defined on $J_{K}^{*}$,

$$
\tilde{\mathcal{H}}^{-1}: J_{K}^{*} \rightarrow J_{K} \text {. }
$$

Similarly to the previous one, we introduce piecewise constants functions $\widetilde{\omega}_{j}(t), \quad t \in[\bar{\alpha}, \bar{\beta})$, $j \epsilon J_{K}$, defined by the equalities

$$
\begin{aligned}
& \widetilde{\omega}_{j}(t)=1 \text { for } t \epsilon\left[\tilde{\xi}_{j}, \tilde{\xi}_{j+1}\right), \\
& \widetilde{\omega}_{j}(t)=0 \text { for } t \in\left[\tilde{\xi}_{j}, \tilde{\xi}_{j+1}\right), j \epsilon J_{K} .
\end{aligned}
$$

System $\widetilde{\omega}(t)=\left\{\widetilde{\omega}_{j}(t)\right\}_{j \in J_{K}}$ of the functions (32) is determined by the grid $\tilde{\Xi}_{K}$. The system is a linearly independent system.

For the space $\widetilde{\Omega}$ we take the space $R^{K}$ of sequences $\tilde{a}=\left(\tilde{a}_{j}\right)_{j \in J_{K}}^{T} \quad$ with the standard scalar product,

$\left(\tilde{a}^{\prime}, \tilde{a}^{\prime \prime}\right)_{\widetilde{\Omega}}=\sum_{i=0}^{K-1} \tilde{a}_{i}^{\prime} \tilde{a}_{i}^{\prime \prime} \quad \forall \tilde{a}^{\prime}, \tilde{a}^{\prime \prime} \in \widetilde{\Omega}$.

Consider a linear space

$$
\begin{aligned}
\tilde{S} & =\left\{\tilde{u}(t) \mid \tilde{u}(t)=\sum_{i \in J_{K}} \tilde{a}_{i} \widetilde{\omega}_{i}(t)\right. \\
\forall \tilde{a} & \left.=\left(\tilde{a}_{i}\right)_{\left\{i \in J_{K}\right\}}^{T} \in \widetilde{\Omega}\right\} .
\end{aligned}
$$

This is the zero-spline space on the grid $\widetilde{\Xi}_{K}$.

\subsection{Projection. Wavelet Decomposition}

Let $\mathcal{P}=\left(p_{r s}\right)_{r \in J_{K}, s \in J_{M}}$ be the matrix of size $K \times M$ with elements

$$
\begin{gathered}
p_{r s}=1 \text { for }\left[\xi_{s}, \xi_{s+1}\right) c\left[\widetilde{\xi}_{r}, \widetilde{\xi}_{r+1}\right), p_{r s}=0 \text { for } \\
{\left[\xi_{s}, \xi_{s+1}\right) \cap\left[\widetilde{\xi}_{r}, \widetilde{\xi}_{r+1}\right)=\emptyset .}
\end{gathered}
$$

In the case under consideration, the relations $\widetilde{\omega}(t) \equiv \mathcal{P} \omega(t) \quad \forall t \in T, \quad \tilde{S} \subset S$ are true.

In the space $S$, consider the system of linear functionals $\left\{\tilde{g}_{j}\right\}_{j \in J_{K}}$ defined by the ratios

$$
<\tilde{g}_{j}, u(\cdot)>=u\left(\widetilde{\xi}_{j}\right) \quad \forall u \in S .
$$

It is easy to see that the system $\left\{\tilde{g}_{j}\right\}_{j \in J_{K}}$ is biorthogonal to the system of $\left\{\widetilde{\omega}_{j^{\prime}}\right\}_{j^{\prime} \epsilon J_{K}}$,

$$
<\tilde{g}_{j}, \widetilde{\omega}_{j \prime}(\cdot)>=\delta_{j, j,} \quad \forall j, j^{\prime} \in J_{K} .
$$

Consider the projective operation $P_{0}: S \rightarrow \tilde{S}$ given by the formula

$$
P_{0}[u(\cdot)](t)=\sum_{j \in J_{K}}<\tilde{g}_{j}, u(\cdot)>\widetilde{\omega}_{j}(t)
$$

Considering that $u(t)=\sum_{s \in J_{M}} c_{S} \omega_{S}(t), \quad$ from (35) we have

$$
\begin{aligned}
P_{0}[u(\cdot)](t)= & \sum_{j \in J_{K}} \sum_{s \in J_{M}} c_{s} \times \\
& <\tilde{g}_{j}, \omega_{s}(\cdot)>\widetilde{\omega}_{j}(t)
\end{aligned}
$$

Introduce the matrix $\boldsymbol{Q}=\left(q_{j s}\right)_{j \in J_{K, S \epsilon J_{M}}}$ of size $K \times M \quad$ with elements $q_{j s}=<\tilde{g}_{j}, \omega_{s}(\cdot)>$, $j \in J_{K}, s \in J_{M}$. From relations (26) -- (29), (33) we have $q_{j s}=\omega_{s}\left(\tilde{\xi}_{j}\right), \quad q_{j s}=1$ for $s=\tilde{\mathcal{H}}(j), q_{j s}=$ 0 for $s \neq \tilde{\mathcal{H}}(j)$, so that

$$
q_{j s}=\delta_{\widetilde{\varkappa}(j), s} .
$$

Ratio (36) can be represented as $P_{0}[u(\cdot)](t)=\sum_{j \epsilon J_{K}} \tilde{a}_{j} \widetilde{\omega}_{j}(t) \quad \forall u \epsilon S$, where $\tilde{a}=\left(\tilde{a}_{j}\right)_{\left\{j \in J_{K}\right\}}^{T}, c=\left(\tilde{c}_{s}\right)_{\left\{s \in J_{M}\right\}}^{T}, \tilde{a}=\mathcal{Q} \mathrm{c}$.

Let us introduce the operator $Q_{0}=I-P_{0}$.

As a result of the projection, we obtain the direct sum (15).

In the case under consideration, the implementation of the decomposition formula is obtained by substituting relations (36) and (37) into representations (10) and (16). Using formulas (36) in representation (18), we obtain the implementation of the reconstruction formulas.

\section{Illustration of Decomposition and Reconstruction}

As an illustration of the previous section's results, we consider the case when $M=6, K=3$. Let us discuss the grid

$$
\Xi_{M}: \bar{\alpha}=\xi_{0}<\xi_{1}<\cdots<\xi_{5}<\xi_{6}=\bar{\beta} .
$$

In this case, we have $J_{6}=\{0,1,2,3,4,5\}$. We should consider six functions $\omega_{j}$ of the form (26) for $j \epsilon J_{6}$. For the space $\Omega$ we take the Euclidean space $R^{6}$ with the standard scalar product, namely for vectors

$$
c^{\prime}=\left(c_{0}^{\prime}, c_{1}^{\prime}, \ldots, c_{5}^{\prime}\right)^{T}, c^{\prime \prime}=\left(c_{0}^{\prime \prime}, c_{1}^{\prime \prime}, \ldots, c_{5}^{\prime \prime}\right)^{T}
$$

with the standard scalar product

$$
\left(c^{\prime}, c^{\prime \prime}\right)_{\Omega}=\sum_{i=0}^{5} c_{i}^{\prime} c_{i}^{\prime \prime} \quad \forall c^{\prime}, c^{\prime \prime} \in \Omega .
$$

Defining the functions $\omega_{j}$ using formula (26),

Consider the space $S=\{u(t) \mid u(t)=$ $\left.\sum_{i=0}^{5} c_{i} \omega_{i}(t) \forall c=\left(c_{i}\right)_{\left\{i \in J_{6}\right\}}^{T} \in \Omega\right\}$.

As $\widetilde{\Xi}_{K}$ consider the grid $\widetilde{\Xi}_{3}$, 
$\widetilde{\Xi}_{3}: \quad \bar{\alpha}=\widetilde{\xi}_{0}<\widetilde{\xi}_{1}<\widetilde{\xi}_{2}<\widetilde{\xi}_{3}=\bar{\beta}$, where $\quad \widetilde{\xi}_{0}=$ $\xi_{0}, \widetilde{\xi}_{1}=\xi_{2}, \widetilde{\xi}_{2}=\xi_{5}, \widetilde{\xi}_{1}=\xi_{6}$.

We have $J_{M}=\{0,1,2,3,4,5\}, J_{K}=\{0,1,2\}, J_{K}^{*}=$ $\{0,2,5\}, \widetilde{\mathcal{H}}(0)=0, \widetilde{\mathcal{H}}(1)=2, \tilde{\mathcal{H}}(2)=5$.

Determining $\widetilde{\omega}_{j}(t)$ by formula (29), consider a linear space

$$
\begin{aligned}
\tilde{S} & =\left\{\tilde{u}(t) \mid \tilde{u}(t)=\sum_{i=0}^{2} \tilde{a}_{i} \widetilde{\omega}_{i}(t)\right. \\
\forall \tilde{a} & \left.=\left(\tilde{a}_{i}\right)_{\left\{i \in J_{3}\right\}}^{T} \in \widetilde{\Omega}\right\} .
\end{aligned}
$$

It is easy to check that

$\widetilde{\omega}_{0}(t)=\omega_{0}(t)+\omega_{1}(t), \widetilde{\omega}_{1}(t)=\omega_{2}(t)+\omega_{3}(t)+$ $\widetilde{\omega}_{4}(t), \widetilde{\omega}_{2}(t)=\omega_{5}(t)$.

Thus $p_{0,0}=p_{0,1}=1, p_{0, j}=0$ for $j \epsilon\{2,3,4,5\}$,

$p_{1,2}=p_{1,3}=p_{1,4}=1, p_{1, j}=0$ for $j \epsilon\{0,1,5\}$,

$p_{2, j}=0$ for $j \epsilon\{0,1,2,3,4\}, p_{2,5}=1$.

According to the formula (36) we have $q_{0,0}=$

$1, q_{0, j}=0$ for $j \epsilon\{1,2,3,4,5\}$,

$q_{1,2}=1, q_{1, j}=0$ for $j \in\{0,1,3,4,5\}$,

$q_{2,5}=1, q_{2, j}=0$ for $j \in\{0,1,2,3,4\}$.

In the case under consideration, the decomposition formulas (15) - (16) take the form $a_{0}=c_{0}, a_{1}=c_{2}, a_{2}=c_{4}, b_{0}=0, b_{1}=-c_{0}+c_{1}$, $b_{2}=0, b_{3}=-c_{2}+c_{3}, b_{4}=-c_{2}+c_{4}, b_{5}=0$.

Since $\mathcal{P}^{T} a=\left(a_{0}, a_{0}, a_{1}, a_{1}, a_{1}, a_{2}\right)^{T}$, and $b_{0}=$ $b_{2}=b_{4}=0$, then the reconstruction formulas (18) can be written as

$$
\begin{gathered}
c_{0}=a_{0}, c_{1}=b_{1}+a_{0}, c_{2}=a_{1}, c_{3}=b_{3}+a_{1}, \\
c_{4}=a_{1}, c_{5}=b_{5}+a_{2} .
\end{gathered}
$$

\section{Zero-type Wavelets in Infinite- Dimensional Spaces}

\subsection{Zero-splines}

On the interval $(\alpha, \beta)$ of the real axis $R^{1}$, we order consider the grid

$$
\Xi: \quad \ldots<\xi_{-2}<\xi_{-1}<\xi_{0}<\xi_{1}<\cdots
$$

with properties

$$
\lim _{j \rightarrow-\infty} \xi_{j}=\alpha, \quad \lim _{j \rightarrow+\infty} \xi_{j}=\beta .
$$

We introduce piecewise constant functions $\omega_{j}(t)$, $\mathrm{t} \epsilon(\alpha, \beta)$, defined by equalities

$\omega_{j}(t)=1$ for $t \in\left[\xi_{j}, \xi_{j+1}\right)$,

$\omega_{j}(t)=0$ for $t \bar{\epsilon}\left[\xi_{j}, \xi_{j+1}\right)$.

System $\omega(t)=\left\{\omega_{j}(t)\right\}_{j \in Z}$ functions (40) are defined by the grid (38) -- (39). This system is a linearly independent system.

For the space $\Omega$, we take the space $l_{2}$ of real sequences

$$
c=\left(\ldots c_{-2}, c_{-1}, c_{0}, c_{1}, c_{2}, \ldots\right)^{T}
$$

with standard scalar product $\left(c^{\prime}, c^{\prime \prime}\right)_{\Omega}=\sum_{i=-\infty}^{+\infty} c_{i}^{\prime} c_{i}^{\prime \prime} \quad \forall c^{\prime}, c^{\prime \prime} \in \Omega$.

Consider a linear space

$S=\left\{u(t) \mid u(t)=\sum_{i=-\infty}^{+\infty} c_{i} \omega_{i}(t) \forall c=\right.$

$\left.\left(c_{i}\right)_{\left\{i \epsilon J_{6}\right\}}^{T} \in \Omega\right\}$.

Note that for fixed $t \in(\alpha, \beta)$ the sequence $\omega(t)$ contains one element equal to the unit. The rest of its elements are equal to zero. So this sequence is an element of the Hilbert space $\Omega$. We can discuss $\omega(t)$ as an abstract function with values in the Hilbert space $\Omega$. That is why the next equivalence is valid $u(t)=\sum_{i=-\infty}^{+\infty} c_{i} \omega_{i}(t) \forall c=\left(c_{i}\right)_{\left\{i \in J_{6}\right\}}^{T} \in \Omega$ $\Leftrightarrow \mathrm{u}(\mathrm{t})=(\mathrm{c}, \omega(t))_{\Omega} \forall c \in \Omega$.

In view of this, formula (42) can be written in the form $S=\left\{u(t) \mid u(t)=(c, \omega(t))_{\Omega} \forall c \in \Omega\right\}$.

\subsection{Embedded Space}

On the same interval $(\alpha, \beta)$, consider the grid

$$
\tilde{\Xi}: \quad \ldots<\tilde{\xi}_{-2}<\tilde{\xi}_{-1}<\tilde{\xi}_{0}<\tilde{\xi}_{1}<\cdots
$$

with properties

$$
\lim _{j \rightarrow-\infty} \tilde{\xi}_{j}=\alpha, \lim _{j \rightarrow+\infty} \tilde{\xi}_{j}=\beta .
$$

Thus, for each $j \in \mathcal{Z}$ we have $\tilde{\xi}_{j} \in \Xi$. Therefore, for each $j \in \mathcal{Z}$ there is a unique $i \epsilon \mathcal{Z}$ such that $\tilde{\xi}_{j}=\xi_{i}$. We denote this mapping $\mathcal{X}$,

$$
\varkappa: Z \rightarrow Z, \quad \mathrm{i}=\varkappa(j), \quad \tilde{\xi}_{j}=\xi_{\varkappa(j) .}
$$

Let $J^{*}$ be the set $\mathcal{X}(Z)$. It is obvious that the single-valued inverse map is defined on $J^{*}$

$$
\mathcal{X}^{-1}: J^{*} \rightarrow Z \text {. }
$$

Similarly to the previous one, we introduce piecewise constants functions

$\widetilde{\omega}_{j}(t), t \epsilon(\alpha, \beta), j \epsilon \mathcal{Z}$, defined by the ratios

$$
\begin{aligned}
& \widetilde{\omega}_{j}(t)=1 \text { for } t \epsilon\left[\tilde{\xi}_{j}, \tilde{\xi}_{j+1}\right), \\
& \widetilde{\omega}_{j}(t)=0 \text { for } t \bar{\epsilon}\left[\tilde{\xi}_{j}, \tilde{\xi}_{j+1}\right), j \in Z .
\end{aligned}
$$

For the space $\widetilde{\Omega}$ we take the space $l_{2}$ of real sequences

$$
\widetilde{c}=\left(\ldots \widetilde{c}_{-2}, \widetilde{c}_{-1}, \widetilde{c}_{0}, \widetilde{c}_{1}, \widetilde{c}_{2}, \ldots\right)^{T}
$$

with standard scalar product

$\left(\tilde{c}^{\prime}, \tilde{c}^{\prime \prime}\right)_{\widetilde{\Omega}}=\sum_{i=-\infty}^{+\infty} \tilde{c}_{i}^{\prime} \tilde{c}_{i}^{\prime \prime} \quad \forall \tilde{c}^{\prime}, \tilde{c}^{\prime \prime} \in \widetilde{\Omega}$.

Consider a linear space

$\tilde{S}=\left\{\tilde{u}(t) \mid \tilde{u}(t)=(\tilde{c}, \widetilde{\omega}(t))_{\widetilde{\Omega}} \forall \tilde{c} \epsilon \widetilde{\Omega}\right\}$.

Thus we have the zero-spline space on the grid $\tilde{\Xi}$.

Let $\mathcal{P}=\left(p_{r s}\right)_{r, s \in \mathcal{Z}}$ be the matrix of infinite size with elements

$p_{r s}=1$ for $\left[\xi_{s}, \xi_{s+1}\right) c\left[\widetilde{\xi}_{r}, \widetilde{\xi}_{r+1}\right), p_{r s}=0$ for $\left[\xi_{s}, \xi_{s+1}\right) \cap\left[\widetilde{\xi}_{r}, \widetilde{\xi}_{r+1}\right]=\emptyset$.

In the case under consideration, the relations $\widetilde{\omega}(t) \equiv \mathcal{P} \omega(t) \quad \forall t \in T, \quad \tilde{S}$ c $S$ are true.

\subsection{Projection onto Embedded Space}

In the space $S$, consider the system of linear functionals $\left\{\tilde{g}_{j}\right\}_{j \in Z}$ defined by the ratios

$$
<\tilde{g}_{j}, u(\cdot)>=u\left(\widetilde{\xi}_{j}\right) \quad \forall u \in S .
$$


It is easy to see that the system $\left\{\tilde{g}_{j}\right\}_{j \in J_{K}}$ biorthogonal to the system of $\left\{\widetilde{\omega}_{j^{\prime}}\right\}_{j^{\prime} \in \mathcal{Z}}$,

$$
<\widetilde{g}_{j}, \widetilde{\omega}_{j \prime}(\cdot)>=\delta_{j, j,}, \quad \forall j, j^{\prime} \in \mathcal{Z} .
$$

Consider the projective operation $P_{0}: S \rightarrow \tilde{S}$ given by the formula

$$
P_{0}[u(\cdot)](t)=\sum_{j \epsilon Z}<\tilde{g}_{j}, u(\cdot)>\widetilde{\omega}_{j}(t) .
$$

Note that there are no questions about the convergence of the sum in (47), because for every fixed $t \in(\alpha, \beta)$ in this sum there is only one nonzero term. Considering that $u(t)=\sum_{s \in Z} c_{s} \omega_{s}(t)$, from (48) we have

$$
\begin{aligned}
P_{0}[u(\cdot)](t)= & \sum_{j \in Z} \sum_{s \in Z} c_{s} \times \\
& <\widetilde{g}_{j}, \omega_{s}(\cdot)>\widetilde{\omega}_{j}(t) \forall u \in S . \text { (48) }
\end{aligned}
$$

We introduce the matrix $\boldsymbol{Q}=\left(q_{j s}\right)_{j, s \in Z}$ of infinite size with elements $q_{j s}=<\tilde{g}_{j}, \omega_{s}(\cdot)>, j, s \in Z$. From relations (40), (43) -- (46) we have $q_{j s}=\omega_{s}\left(\tilde{\xi}_{j}\right)$, $q_{j s}=1$ for $s=\varkappa(j), q_{j s}=0$ for $s \neq \varkappa(j)$, so that $q_{j s}=\delta_{\varkappa(j), s}$. Ratio (48) can be represented as

$$
P_{0}[u(\cdot)](t)=\sum_{j \in Z} \tilde{a}_{j} \widetilde{\omega}_{j}(t) \quad \forall u \in S,
$$

where $\tilde{a}=\left(\tilde{a}_{j}\right)_{\{j \in Z\}}^{T}, c=\left(\tilde{c}_{s}\right)_{\{s \in Z\}}^{T}$,

$$
\tilde{a}=Q \text { c. }
$$

\subsection{Wavelet Decomposition}

Let us introduce the operator $Q_{0}=I-P_{0}$. As a result of projection (47), we obtain the direct sum $S=\tilde{S}+W$, where $\tilde{S}=P_{0} S, W=Q_{0} S$.

We introduce the notation

$$
b=c-\mathcal{P}^{*} \boldsymbol{Q} c .
$$

By Theorem 3, we obtain ratios

$$
\begin{gathered}
Q_{0}[u(\cdot)](t)=u(t)-P_{0}[u(\cdot)](t)=(b, \omega)_{\Omega}, \\
\mathrm{c}=\mathcal{P}^{*} \tilde{a}+b .
\end{gathered}
$$

Formulas (50) - (52) illustrate the wavelet decomposition.

Thus, the initial flow $c \in \Omega$ spawns two flows: main flow $\tilde{a}$ and wavelet flow $b$. Relations (50) (51) are the decomposition formulas. Ratio (52) is the reconstruction formula.

\section{About Continual Wavelets}

\subsection{Generating Function}

To illustrate the possibilities of the proposed approach, consider a continuous wavelet decomposition.

Let $\mathcal{M}$ be the Lebesgue measurable set of the real axis $R^{1}$. We discuss point $t$ of an interval $(\alpha, \beta) c R^{1}$.

In the case of measurable sets of the symbol " $\forall x \in \mathcal{M}^{\prime \prime}$, we agree on use with the meaning "for almost all $x \in \mathcal{M}$ ". $\quad$ For example, the expression " $f(x)=0 \quad \forall x \in \mathcal{M} "$ means that the function $f(x)$ is equal to zero at all points of the set $\epsilon \mathcal{M}$, excluding some set of measure zero.

Consider the function $\omega(x, t), t \in(\alpha, \beta), x \in \mathcal{M}$. We suppose that for each fixed $t \in(\alpha, \beta)$ the element $\omega(\cdot, t)$ belongs to the space $L_{p}(\mathcal{M})$. Here $p$ is fixed number, $p>1$. In this way, $\omega(t)=\omega(\cdot, t)$, $t \in(\alpha, \beta)$, is a trajectory in the space $L_{p}(\mathcal{M})$. In what follows we assume that the functions depending on $t$ are defined for all points $t$ of the interval $(\alpha, \beta)$.

According to the general scheme (see Section 2.) we put $\Omega=L_{p}(\mathcal{M}), \quad \Omega^{*}=L_{q}(\mathcal{M})$, where $q^{-1}=1-$ $p^{-1}$.

Consider linear space $S$ defined by the relation $S=\left\{u(t) \mid u(\mathrm{t})=\int_{\mathcal{M}} c(x) \omega(x, t) d x \forall c \in L_{q}(\mathcal{M})\right\}$.

Remark 1. The zero-order wavelets considered in the previous section are a special case of the situation under consideration here. In particular, using grid (38) - (39), we can define the function $\omega(t)=\omega(\cdot, t)$ in the following way. For each $j \in Z$ and $x \in\left[\xi_{j}, \xi_{j+1}\right)$ we put $\omega(x, t)=1$ for $t \epsilon\left[\xi_{j}, \xi_{j+1}\right), \omega(x, t)=0$ for $t \bar{\epsilon}$ $\left[\xi_{j}, \xi_{j+1}\right), S=\left\{u(t) \mid u(t)=(c, \omega(t))_{\Omega} \forall c \in \Omega^{*}\right\}$.

\subsection{Embedded Space}

Consider a measurable set $\mathcal{K}$ contained in $\mathcal{M}$, $\mathcal{K} \subset \mathcal{M}$. Consider the function $\widetilde{\omega}(y, t)$,

$\mathrm{t} \epsilon(\alpha, \beta), y \in \mathcal{K}$, which for each fixed $\mathrm{t} \in(\alpha, \beta)$ is an element of the space $L_{p}(\mathcal{K})$. Thus $\widetilde{\omega}(t)=\widetilde{\omega}(\cdot, t)$, $\mathrm{t} \epsilon(\alpha, \beta)$, is a trajectory in the space $L_{p}(\mathcal{K})$. In accordance with the general scheme, we put $\widetilde{\Omega}=$ $L_{p}(\mathcal{K}), \widetilde{\Omega}^{*}=L_{q}(\mathcal{K})$.

Let the linear space $\widetilde{S}$ be defined by $\widetilde{S}=$ $\left\{\tilde{u}(t) \mid \tilde{u}(t)=\int_{\mathcal{K}} \tilde{a}(y) \widetilde{\omega}(y, t) d y \forall \tilde{a} \epsilon L_{q}(\mathcal{K})\right\}$.

Consider the function $\tilde{P}(y, x), y \in \mathcal{K}, x \in \mathcal{M}$, with properties $\tilde{P}(y, \cdot) \in L_{q}(\mathcal{M}) \forall y \in \mathcal{K}$, $\widetilde{P}(\cdot, x) \in L_{p}(\mathcal{K}) \forall x \in \mathcal{M}$.

We suppose that $\widetilde{\omega}(y, t)=$

$=\int_{\mathcal{M}} \tilde{P}(y, x) \omega(x, t) d x \forall t \epsilon(\alpha, \beta), \forall y \in \mathcal{K}$.

It follows from (53) that the operator $\mathcal{P}: \Omega \rightarrow \widetilde{\Omega}$, defined by the formula

$[\mathrm{v}(\cdot)](y)=\mathcal{P}[\mathrm{v}(\cdot)](y)=$ $\int_{\mathcal{M}} \tilde{P}(y, x) v(x) d x \quad \forall y \in \mathcal{K} \forall v \in \Omega$,

has the property

$$
\widetilde{\omega}(t)=\mathcal{P} \omega(t) .
$$

In accordance with Theorem 1, relation (54) implies embedding of the space $\widetilde{S}$ into the space $S$, 
$\widetilde{S} \mathrm{cS}$.

In what follows, we consider the projection of the space $S$ onto $\widetilde{S}$.

\subsection{Embedded Space Projection}

As an illustration, we consider a simple example of a projection operation from the space $S$ to the space $\widetilde{S}$.

In what follows, we use the concept of the restriction of a function from the set $\mathcal{M}$ to the set $\mathcal{K}$. The restriction of the function $v(x)$ from $x \in \mathcal{M}$ to $\mathcal{K}$ is called function $\tilde{v}(x)$ such that $\tilde{v}(x)=v(x)$ $\forall x \in \mathcal{K}$, and $\tilde{v}(x)=0 \quad \forall x \in \mathcal{M} \backslash \mathcal{K}$.

The restriction of $v(x)$ from $x \in \mathcal{M}$ to is denoted by the symbol $\left.\right|_{\mathcal{K}}$. In this way, we have $\tilde{v}(x)=\left.v\right|_{\mathcal{K}}(x)$.

We propose that functions $f \in L_{q}(\mathcal{K})$ are propagated from $\mathcal{K}$ onto $\mathcal{M}$ by zero. Therefore, we can discuss the embedding $L_{q}(\mathcal{K}) c L_{q}(\mathcal{M})$.

Let $\mathcal{P}$ be the operation of the restriction from the set $\mathcal{M}$ onto the set $\mathcal{K}$,

$$
\begin{aligned}
& \mathcal{P}[v(\cdot)](x)=\left.v\right|_{\mathcal{K}}(x) \quad \forall v \in \Omega \quad \forall x \in \mathcal{M} . \\
& (\tilde{a}, \mathcal{P} v)_{\widetilde{\Omega}}=\left(\mathcal{P}^{*} \tilde{a}, v\right)_{\Omega} \quad \forall \tilde{a} \in \widetilde{\Omega}^{*} \quad \forall v \in \Omega
\end{aligned}
$$

In the case under consideration, the operation $\mathcal{P}$ the restriction to $\mathcal{K}$. Therefore, formula (57) takes the form

$$
\int_{\mathcal{K}} \tilde{a}(y) v(y) d y=\int_{\mathcal{M}}\left(\mathcal{P}^{*} \tilde{a}\right)(x) v(x) d x
$$

$\forall \tilde{a} \in \widetilde{\Omega}^{*} \forall v \in \Omega$.

By (58) we see that the values of $v(x)$ on the set $\mathcal{M} \backslash \mathcal{K}$ does not play a role. So in view of the arbitrariness of $v \in \Omega=L_{q}(\mathcal{M})$, the integral on the right parts of the above equality can be calculated only over the set $\mathcal{K}$. We have

$$
\int_{\mathcal{K}} \tilde{a}(y) v(y) d y=\int_{\mathcal{K}}\left(\mathcal{P}^{*} \tilde{a}\right)(x) v(x) d x
$$

$\forall \tilde{a} \in \widetilde{\Omega}^{*} \forall v \in \Omega$.

Identity (59) implies the formula

$$
\begin{aligned}
& \left(\mathcal{P}^{*} \tilde{a}\right)(x)=\tilde{a}(x) \text { for } x \in \mathcal{K}, \\
& \left(\mathcal{P}^{*} \tilde{a}\right)(x)=0 \text { for } x \in \mathcal{M} \backslash \mathcal{K}
\end{aligned}
$$

We suppose that

$$
\widetilde{\omega}(t)=\mathcal{P} \omega(t)
$$

By (61) formula (55) follows from Theorem 1 and relation (56).

To define the operation $P_{0}$, we consider an arbitrary element $u \in S$. By definition the space $S$ has the form $u(t)=\int_{\mathcal{M}} c(x) \omega(x, t) d x$, where the function $c(x)$ lies in the space $\Omega^{*}=L_{q}(\mathcal{M})$.

Obviously narrowing $\left.c\right|_{\mathcal{K}}$ of this function to the set $\mathcal{K}$ lies in the space $\widetilde{\Omega}^{*}=L_{q}(\mathcal{K})$.
By the image of the function $u(t)$ under the mapping $P_{0} \quad$ we consider the function $\tilde{u}(t)=$ $\int_{\mathcal{K}} \tilde{a}(y) \widetilde{\omega}(y, t) d y$, where $\tilde{a}(y)=\left.c\right|_{\mathcal{K}}(y)$.

In the previously adopted notation, this can be written with the ratio

$P_{0}\left[\left(c, \omega(\cdot)_{\Omega}\right](t)=(\tilde{a}, \widetilde{\omega}(t))_{\widetilde{\Omega}} \forall c \in \Omega^{*} \forall t \epsilon(\alpha, \beta)\right.$, where $\tilde{a}(y)=\left.c\right|_{\mathcal{K}}(y) \forall y \in \mathcal{K}$.

A shorter record of the last relation is

$P_{0}\left[\left(c, \omega(\cdot)_{\Omega}\right](t)=\right.$ $=\left(\left.c\right|_{\mathcal{K}}, \widetilde{\omega}(t)\right)_{\widetilde{\Omega}} \forall c \in \Omega^{*} \forall t \epsilon(\alpha, \beta)$.

Obviously, the operator $P_{0}$, defined by formula (62), is linear operator. The last one acts from $S$ to $\widetilde{S}$. Let us show that the operator $P_{0}$ is idempotent, that is, the relation $P_{0}^{2}=P_{0}$ is true. It suffices to establish that on the space $\widetilde{S}$ the operator acts as the identity operator. Indeed, according to the definition of the operator $P_{0}$, we have

$$
\begin{aligned}
& P_{0}\left[\int_{\mathcal{M}} c(x) \omega(x, \cdot) d x\right](t)= \\
& \quad=\int_{\mathcal{K}} c(y) \widetilde{\omega}(y, t) d y \forall c \in \Omega^{*} \forall t \in(\alpha, \beta) .
\end{aligned}
$$

Instead of $c(x)$, we substitute the function $\tilde{a}(x)=\left.c\right|_{\mathcal{K}}(x)$. Now the integral on the left-hand side of formula (63) can be calculated only over the domain $\mathcal{K}$, so we have

$$
\begin{aligned}
& P_{0}\left[\int_{\mathcal{K}} \tilde{a}(x) \omega(x, \cdot) d x\right](t)= \\
& \quad=\int_{\mathcal{K}} \tilde{a}(y) \widetilde{\omega}(y, t) d y \forall c \epsilon \Omega^{*} \forall t \epsilon(\alpha, \beta) .
\end{aligned}
$$

Taking into account relation (61), we arrive at the identity $P_{0} \tilde{u}=\tilde{u} \quad \forall \tilde{u} \in \tilde{S}$. Thus, the operator $P_{0}$ is the projector.

From formulas (12) and (63) in the case under consideration, we obtain

$$
\begin{aligned}
& \int_{\mathcal{K}}(\boldsymbol{Q} \mathrm{c})(y) \widetilde{\omega}(y, t) d y= \\
& \quad=\int_{\mathcal{K}} c(y) \widetilde{\omega}(y, t) d y \forall c \in \Omega^{*} \forall t \in(\alpha, \beta) .
\end{aligned}
$$

Using the completeness of the abstract function $\widetilde{\omega}(t)$, we arrive at equality

$$
(\boldsymbol{Q} \mathrm{c})(y)=\left.c\right|_{\mathcal{K}}(y) \quad \forall y \in \mathcal{K} .
$$

\subsection{Wavelet Decomposition}

Let's introduce the operator $Q_{0}=I-P_{0}$. As a result of projection (64), we obtain the direct sum $S=\tilde{S}+W$, where $\tilde{S}=P_{0} S, W=Q_{0} S$. Let us turn to the decomposition formulas (10), (16) and (65) in the case under consideration.

The main flow is obtained by the formula $\tilde{a}=\mathcal{Q} c \Leftrightarrow \tilde{a}=\left.c\right|_{\mathcal{K}} \Leftrightarrow \tilde{a}(y)=c(y) \quad \forall y \in \mathcal{K}$.

So in this case of main flow $\tilde{a}(y)$ is determined by the values of the original flow $c(y)$ on the set $y \in \mathcal{K}$,

$$
\tilde{a}(y)=c(y) \quad \forall y \in \mathcal{K} .
$$

The wavelet flow is obtained according to the relations

$$
\begin{gathered}
b=c-\mathcal{P}^{*} \boldsymbol{Q}\left(\left.c\right|_{\mathcal{K}}\right) \Leftrightarrow \\
\Leftrightarrow b(x)=c(x)-\mathcal{P}^{*} \boldsymbol{Q}\left(\left.c\right|_{\mathcal{K}}\right)(x) \forall x \in \mathcal{M}
\end{gathered}
$$


Using formula (60), we find

$b(x)=c(x)-\left(\left.c\right|_{\mathcal{K}}\right)(x)=0$ for $x \in \mathcal{K}$,

$b(x)=c(x)-0=c(x)$ for $x \in \mathcal{M} \backslash \mathcal{K}$.

Thus we obtain decomposition formulas (66) (67).

For the formula (18), we take into account relation (60), (67).

We have

$$
\begin{aligned}
& c(x)=b(x)+\mathcal{P}^{*} \tilde{a}(x) \Leftrightarrow \\
& c(x)=0+\tilde{a}(x) \text { for } x \in \mathcal{K} \text {, } \\
& c(x)=b(x)+\mathcal{P}^{*} \tilde{a}(x) \Leftrightarrow \\
& c(x)=b(x)+0 \text { for } x \in \mathcal{M} \backslash \mathcal{K} \text {. } \\
& \text { Therefore we find } \\
& c(x)=\tilde{a}(x) \text { for } x \in \mathcal{K} \text {, } \\
& c(x)=b(x) \text { for } x \in \mathcal{M} \backslash \mathcal{K} \text {. }
\end{aligned}
$$

Ratios (68) are reconstruction formulas in the discussed case.

\section{Conclusion}

In this paper, a new approach to the construction of wavelet expansions is considered. This approach allows us to consider discrete and continuous flows of a various nature. Thanks to this approach, it is possible to consider discrete numerical flows, flows of matrices, flows of p-adic numbers, etc.

The possibility of wavelet decomposition of continuous streams seems to be very important, which makes it possible to include in the processing not only discrete (in particular, digital) signals, but also streams representing analog signals. The result obtained here can be viewed, on the one hand, as the limiting closure of the algorithm (in the sense of S.L. Sobolev), and on the other hand, as a source of discrete wavelet approximations (see Remark 1.)

The need for a very brief presentation of the material forced the authors to limit themselves to the simplest examples illustrating the proposed approach. However, the examples provided suggest broad application prospects. The authors intend to investigate some of these applications in the future.

\section{References:}

[1] Mozhde Firoozi Pouyani, Mansour Vali, Mohammad Amin Ghasemi, Lung sound signal denoising using discrete wavelet transform and artificial neural network, Biomedical Signal Processing and Control. Vol.72, 2022, article id.103329.

[2] Fakharian P., Naderpour, H. Damage Severity Quantification Using Wavelet Packet Transform and Peak Picking Method,
Practice Periodical on Structural Design and Construction. Vol. 27(1), 2022, article id. 04021063.

[3] Ramalingappa, L., Manjunatha, A. Power quality event classification using complex wavelets phasor models and customized convolution neural network, International Journal of Electrical and Computer Engineering. Vol. 12(1), 2022, pp. 22-31.

[4] Daubechies I. Ten Lectures on Wavelets, CBMS-NSR, Series in Appl. Math.SIAM,1992.

[5] Chui C.K. An Introduction to Wavelets.-N.Y.: Academic Press, 1992.

[6] Stephane Mallat. A Wavelet Tour of Signal Processing.-N.Y.: Academic Press, 1999.

[7] Yu.N. Subbotin, N. I. Chernykh, Interpolation wavelets in boundary value problems, Proc. Steklov Inst. Math. (Suppl.). Vol. 300, 2018, pp. 172-183.

[8] Novikov I.Ya.,Protasov V.Yu., Skopina M.A. Wavelet Theory. AMS, Translations Mathematical Monographs. V. 239 (2011).

[9] Boris Shumilov. Shifted Cubic Spline Wavelets with Two Vanishing Moments on the Interval and a Splitting Algorithm, WSEAS Transactions on Systems, Vol. 19, 2020, pp.149-158.

[10] Boris Shumilov. On Five-Diagonal Splitting for Cubic Spline Wavelets with Six Vanishing Moments on a Segment, WSEAS Transactions on Information Science and Applications. Vol. 17, 2020, pp. 156-165.

[11] Ana Benavente, Ole Christensen, Marzieh Hasannasab, Hong Oh Kim, Rea Young Kim, Federico D.Kovac, Approximately dual pairs of wavelet frames, Journal of Mathematical Analysis and Applications. Vol. 507(2), 2022, article id.125841.

[12] Abdullah, M.K., Kamyabi-Gol, R.A. On wavelet multiplier and Landau-Pollak-Slepian operators on L2(G, H), Journal of PseudoDifferential Operators and Applications. Vol. 10(2), 2019, pp.257-267.

[13] Kaur, N., Goyal, K. An adaptive wavelet optimized finite difference B-spline polynomial chaos method for random partial differential equations, Applied Mathematics and Computation. Vol.415, 2022, article id.126738.

[14] Huang, C.-S., O'Hara, J.G., Mataramvura, S. Highly efficient Shannon wavelet-based pricing of power options under the double exponential jump framework with stochastic jump intensity and volatility, Applied 
Mathematics and Computation, Vol.414, February 2022, article id. 126669.

[15] Yahia, S., Said, S., Zaied, M. Wavelet extreme learning machine and deep learning for data classification, Neurocomputing, Vol.470, 2020, pp. 280-289.

[16] Zhou, X. Uniform convergence rates for wavelet curve estimation in sup-norm loss, Journal of Computational and Applied Mathematics, Vol. 400,2021, article id. 113752

[17] Kadri Harouna, S., Perrier, V. Homogeneous Dirichlet wavelets on the interval diagonalizing the derivative operator, and application to free-slip divergence-free wavelets. Journal of Mathematical Analysis and Applications 505(2), 2019, article id. 125479.

[18] Kumar, R., Nigam, R., Singh, S.K. Selection of suitable mother wavelet along with vanishing moment for the effective detection of crack in a beam, Mechanical Systems and Signal Processin. Vol.163,2022, article id.108136.

[19] Wang, Z., Wang, C., Ding, L., Wang, Z., Liang, S. Parameter identification of fractional-order time delay system based on Legendre wavelet, Mechanical Systems, and Signal Processing. Vol. 163,2022, article id. 108141.

[20] Yuri K. Dem'yanovich, Tatjana A. Safonova, Andrey N. Terekhov, Approximation Functionals and Their Application, WSEAS Transactions on Mathematics. Vol. 20, 2021, pp. 489-495.

\section{Creative Commons Attribution License 4.0}

(Attribution 4.0 International, CC BY 4.0)

This article is published under the terms of the Creative Commons Attribution License 4.0

https://creativecommons.org/licenses/by/4.0/deed.en US 